\title{
Nasal obstruction as a key symptom in allergic rhinitis: efficacy and safety of a medical device in children
}

\author{
Desiderio Passali ${ }^{1, *}$, Luisa Maria Bellussi ${ }^{1}$, Dario Gregori ${ }^{2}$, Maria Lauriello ${ }^{3}$, \\ Francesco Maria Passali ${ }^{4}$, Giulio Cesare Passali ${ }^{5}$ \\ Gip Stop Study Group ${ }^{a}$ \\ ${ }^{1}$ Department of Otolaryngology, University of Siena, Italy \\ ${ }^{2}$ Laboratories of Epidemiological Methods and Biostatistics, Department of Environmental Medicine and Public Health, University of Padova Italy \\ ${ }^{3}$ Experimental Medicine Department University of L'Aquila, Italy \\ ${ }^{4}$ Department of Otorhinolaryngology, "Tor Vergata" University, Rome, Italy \\ ${ }^{5}$ ENT Clinic Catholic University of the Sacred Heart Medical School: Roma Italy
}

\section{A R T I C L E I N F O}

Article history:

Received: 11.01.2012

Accepted: 29.02.2012

Published on line: 24.05.2012

Keywords:

- Allergic Rhinitis

- Medical Device

- VAS

- Nasal Obstruction

- Nasal Congestion

- HMGB1 protein

\begin{abstract}
A B S T R A C T
Background: Allergic rhinitis (AR) is a global health problem that has increased rapidly in prevalence over the past few decades. Nasal congestion, which represents a cardinal symptom of AR, appears to be difficult to treat and, especially for pediatric population, therapies that are both well tolerated and effective in relieving nasal congestion are needed. The aim of the present study was to evaluate the safety and clinical effectiveness of the medical device (lactoferrin, carboximetil $\beta$-glucan, D-panthenol, dipotassium glycyrrhizinate) in reducing the allergic rhinitis symptoms. Methods: A multicenter prospective study with a pre-post design was performed consecutively enrolling 100 pediatric patients of both genders affected by persistent AR. Patients received 2 puffs into each nostril 2 times a day over the course of 4 weeks. The severity of AR symptoms, as measured by VAS score, was assessed before and after treatment. The main outcomes were improvement in each symptom score and in overall symptom burden (as measured by average symptom scores). Differences in symptoms scores measured before and after treatment were compared using paired-sample Wilcoxon signed rank test. The proportion of participants with adverse effects attributed to the treatment was also computed. Results: All considered symptoms, including nasal congestion, significantly improve after treatment $(p<0.001)$, while only 9 patients suffered adverse effects (itch, burning sensation, dryness). Conclusion: These results confirm the efficacy and safety of this medical device in pediatric population
\end{abstract}

(C) 2012 Polish Otorhinolaryngology - Head and Neck Surgery Society. Published by Elsevier Urban \& Partner Sp. z o.o. All rights reserved.

\footnotetext{
* Corresponding author: Ear, Nose, and Throat Clinic, Policlinico Le Scotte, University of Siena, Italy, Via Anagnina 718,00118 Rome, Italy. Tel.: +393356102667 ; fax: +390679844154 .

E-mail addresses: d.passali@virgilio.it, desiderio@passali.org (D. Passali).

a Grip Stop Study Group: Raffaele Fiorella: ENT Department University of Bari medical School, Bari Italy. Pasquale Cassano: ENT Department University of Foggia Medical School, Foggia Italy. Angelo Camaioni: ENT Clinic S. Giovanni Addolorlata Hospital Rome, Italy. Salvatore Restivo: ENT Department University of Palermo Medical School, Palermo Italy. Marco Piemonte: ENT Clinic Ospedali Riuniti di Udine, Italy. Michele De Benedetto: ENT Clinic Tito Fazzi Ospital Lecce, Italy. Giuseppe Caruso: Department of Otolaryngology, University of Siena, Italy. Fabrizio Ottavani: Department of Otorhinolaryngology, "Tor Vergata" University, Rome, Italy.

0030-6657/\$ - see front matter @ 2012 Polish Otorhinolaryngology - Head and Neck Surgery Society. Published by Elsevier Urban \& Partner Sp. z o.o. All rights reserved. http://dx.doi.org/10.1016/j.otpol.2012.05.019
} 


\section{Introduction}

Allergic rhinitis (AR) is a global health problem that has increased rapidly in prevalence over the past few decades [1]. The disease is now expected to affect more than 500 million individuals worldwide. Particularly, it is the most common chronic condition in children being estimated to affect up to $40 \%$ of all children. It is usually diagnosed by the age of 6 years [2], and its symptoms, including nasal congestion, rhinorrhea, sneezing and non-nasal symptoms such as burning, itching and watery eyes or itching ears and palate, have a major impact on the quality of life and school productivity, as recently outlined by the World Health Organization in the Allergic Rhinitis and its Impact on Asthma workshop [3]. Especially, AR has profound effects on school absenteeism, since symptoms during the day can hamper concentration and lead to learning problems, while nocturnal symptoms can cause sleep loss and secondary daytime fatigue, further undermining child's ability to function well during the school day [4]. Moreover, individuals with rhinitis find it socially embarrassing to be seen sneezing, sniffing, or blowing their nose.

Effective management of AR often requires a combination of pharmacologic (including corticosteroids antihistamines, decongestants, antihistamine-decongestant combinations) and non-pharmacologic interventions such as nasal irrigation. However, nasal congestion which represents a cardinal symptom of AR, appears to be difficult to treat. Recently Nathan [5] systematically reviewing strengths and weaknesses of available treatments, as reported in the literature, found that therapy for nasal congestion in AR is often hampered by limitations associated with individual agents; for example, decongestants are effective in the control of nasal congestion, but their use is restricted by their adverseevent profiles, concluding that there is a need for therapies that are both well tolerated and effective in relieving nasal symptoms in AR.

Recently the importance of the HMGB1 protein in the pathogenesis of several inflammatory diseases has been demonstrated. This protein is released from necrotic or immune-activated cells and, acting on specific membrane receptors, causes: the release of pro-inflammatory mediators, the endothelial activation, and the survival of inflammatory cells, mainly eosinophils [6]. The discovery of HMGB1 protein in nasal secretion of patients with allergic and non-allergic inflammation of the nose and paranasal sinuses may contribute to the inflammatory process becoming chronic. As a consequence, the inhibition of HMGB1 protein may be an efficacious and innovative therapeutic strategy for patients with chronic rhinosinusitis.

Glycyrrhizin, a natural triterpene glycoconjugate derived from the root of licorice (Glycyrrhiza glabra) inhibits the chemotactic and mitogenic functions of HMGB1 protein interfering with the inflammatory process. The glycyrrhizininduced inhibition of the physiological activities of HMGB1 may be involved in the anti-inflammatory effect of glycyrrhizin in vivo.

The interaction between glycyrrhizin and HMGB1 is limited to a scavenger function of the inflammatory protein released by the necrotic cells [7]. Carboxymethyl $\beta$-glucan has decongestant and immunostimulating effects on the nasal mucosa [8]. Therefore, the present study was conducted in order to evaluate the safety and clinical effectiveness of a medical device (lactoferrin, carboximetil $\beta$-glucan, $D$-panthenol, dipotassium glycyrrhizinate) in reducing allergic rhinitis symptoms including nasal obstruction, rhinorrhea, sneezing and watery eyes as measured by a 0 to 5 visual analog scale (VAS). According to the EC Directive 93/42 "medical device" means "any instrument, apparatus, applance, software, material or other article [...] which does not achieve its principal intended action in or on human body by pharmacological, immunological or metabolic means [.. .]"

It seems likely that glycyrrhizin may exert its antiinflammatory effect in a manner similar to steroid antiinflammatory drugs, because glycyrrhizin is a steroid-like molecule and directly binds to the glucocorticoid-like receptor [7]. Besides, glycyrrhizin has a scavenger function of the inflammatory protein HMGB1 released by the necrotic cells [8].

Carboxymethyl $\beta$-glucan, being a natural polysaccharide, acts on the solution viscosity, thus increasing its cleansing power. Besides, it has decongestant and immunostimulating effects on the nasal mucosa [9]. D-panthenol is used in gel preparations to reduce crusting and discomfort in the postoperative period of endonasal surgery [10].

Lactoferrin and lysozyme are antimicrobial peptides and as such, an important component of the innate immune system. They offer the body a first-line defense against a wide range of invading pathogens. In addition lactoferrin is the human body's only known antimicrobial peptide with antibiofilm properties [11, 12].

As a whole, the study device presents different mechanisms of action on nasal obstruction as main and troublesome symptom of allergic rhinitis in children. It has anti-inflammatory and cleansing effects protecting the mucosa at the same time from viral and bacterial superinfection and restoring the normal cytological architecture.

\section{Methods}

\section{Study design}

A multicenter prospective study with a pre-post design was performed consecutively on 100 pediatric patients (males 58\%) aged from 8 to 14 years (mean age 9.5), enrolling in 5 Italian Otolaryngology Departments, affected by persistent mild-tomoderate allergic rhinitis diagnosed according to the ARIA criteria: intermittent AR is defined as experiencing symptoms for $<4$ days/wk or $<4$ consecutive weeks; persistent AR is termed as symptoms occurring for more than 4 days/wk and more than 4 consecutive weeks; in mild form symptoms are present but they do not interfere with daily activities.

Exclusion criteria were the presence of acute upper respiratory infections and the use, during the 4 preceding weeks, of nasal or oral corticosteroids or decongestants, and antileukotrienes and antihistamines during the previous week.

In accordance with the study protocol, patients received 2 puffs into each nostril 2 times a day over the course of 4 weeks. Patients visited the investigators twice during the study period; at the enrolment time and at the end of the 
$\mathrm{x}$ week. The severity of AR symptoms was assessed during each visit, whereas the tolerability of the product was assessed at the second visit.

VAS was used to quantify the subjective feeling of nasal obstruction, rhinorrhea, sneezing and watery eyes. The subjective symptom score was obtained with a visual analog scale modified from Eccles' model [9]. Patients rated the perceived degree of their obstruction on a scale of 0 (complete patency) to 5 (complete stenosis). Likewise, VAS was used for other symptoms. Ethics committee approval and informed consent from the parents of both children were obtained.

\section{Sample size}

The primary outcomes of the present study were symptom resolution (improvement in each symptom score from enrolment to week $\mathrm{x}$ ) and improvement in overall symptom burden (as measured by average symptom scores). The proportion of participants with adverse effects attributed to the treatment was also computed. The study was designed as a pre-post study, comparing the evolution of the VAS over 2 time intervals. The targeted Delta over time in VAS was set at 1 , with an expected standard deviation of 1.5 , compared to a no-change null hypothesis. Choosing an alpha-level of 0.05 and a power of $0.80,18$ patients were computed as needed for detecting such Delta.

\section{Statistical analysis}

Continuous variables were always expressed as median and interquartile difference and categorical variables as percentages and absolute numbers. Differences between symptoms felt before and after Grip Stop treatment were compared using paired-sample Wilcoxon signed rank test.

\section{Results}

Table I presents subjective evaluation of symptoms before and after treatment: all symptoms, including nasal congestion, significantly improve after treatment $(p<0.001)$. Overall symptom burden before and after is also reported:

The proportions of patients referring the occurrence of adverse effects during treatment with the nasal device in

\begin{tabular}{|c|c|c|c|}
\hline & \multicolumn{2}{|c|}{ VAS } & \multirow[t]{2}{*}{$\mathrm{p}$} \\
\hline & $\begin{array}{c}\text { before } \\
(\mathrm{N}=100)\end{array}$ & $\begin{array}{c}\text { after } \\
(\mathrm{N}=100)\end{array}$ & \\
\hline Nasal obstruction & $4 / 5 / 5$ & $1 / 2 / 3$ & $<0.001$ \\
\hline Sneezing & $1 / 2 / 3$ & $0 / 1 / 2$ & $<0.001$ \\
\hline Watery eyes & $0 / 1 / 2.25$ & 0/0/1 & $<0.001$ \\
\hline Rhinorrhea & $3 / 4 / 5$ & $1 / 1 / 2$ & $<0.001$ \\
\hline Overall symptom burden & $4 / 5 / 5$ & $1 / 2 / 3$ & $<0.001$ \\
\hline
\end{tabular}

Numbers are I quartile/Median/III quartile. P-value refers to a significantly different distribution of each given variable before and after treatment. study have been recorded: only 9 (0.09\%) patients suffered from adverse effects. Particularly 2 subjects complained of burning sensation, 2 of dryness, and 5 of itching.

\section{Discussion}

Allergic rhinitis is an antigen-mediated inflammation of the nasal mucosa that may extend into the paranasal sinuses and the middle ear. The principles of treatment are the same for children and adults, but special care has to be taken to avoid the side effects. In fact, during infancy and childhood medication absorption distribution, metabolism and excretion are profoundly affected, in turn, affecting optimal dosing, efficacy, and safety. Many medications currently prescribed for adults (i.e., systemic or topical decongestants) in our country lack paediatric approval. Non-pharmacologic treatment of allergic rhinitis in children involves educating the family and the child about the nature of the disease, avoiding allergen triggers and respiratory tract irritants, the most important of which is tobacco smoke.

Of the available treatment options for pediatric allergic rhinitis, intranasal corticosteroids and the newer oral antihistamines are first-line treatments.

Intranasal corticosteroids are the most effective antiinflammatory agents used for the treatment of allergic rhinitis. They control itching, sneezing, rhinorrhea, and stuffiness in most patients, but do not alleviate ocular symptoms. The safety implications associated with corticosteroids are long-term, dose-related systemic effects, such as suppression of adrenocortical function, growth, and bone metabolism. The extent of these effects is influenced by a number of factors including corticosteroid type, pharmacokinetic profile, mode of delivery, and delivery device [10]. Long-term growth studies in children using fluticasone, mometasone, and budesonide have shown reassuring safety data, unlike beclomethasone [11]. Nevertheless, growth should be regularly monitored in children receiving intranasal corticosteroids [7].

Second-generation antihistamines have become increasingly popular because of their comparable efficacy and lower incidence of adverse effects relative to their firstgeneration counterparts which are associated with unwanted adverse effects such as cardiotoxicity, sedation and impairment of psychomotor function [10]. The newer antihistamines, developed with the aim of being more specific for the histamine $\mathrm{H}$ [1] receptor and of overcoming these adverse effects, are clinically efficacious for alleviating symptoms of allergic rhinitis that are attributed to the earlyphase reaction, such as rhinorrhea, pruritus, and sneezing. However, they have demonstrated poor efficacy in controlling nasal congestion, which is related to the late-phase reaction. Therefore, the use of antihistamines in combination with a decongestant may be required to control the complete range of AR symptoms, including more severe nasal congestion [12].

Decongestants are sympathomimetic drugs that constrict capacitance vessels in the turbinates and decrease nasal congestion. Due to their vasoconstrictor action, the sympathomimetic decongestants oppose vasodilation, reducing nasal airway resistance and thus facilitating nose breathing [13]. 
Systemic and nasal decongestants are available. Systemic nasal decongestants are indicated for temporary relief of nasal congestion, to promote nasal or sinus drainage, and for cough caused by postnasal drip. Topical nasal decongestants are indicated for the symptomatic relief of both nasal and nasopharyngeal mucosal congestion. Side effects from decongestants are more likely to occur in children than in adults. The effects include elevated blood pressure, tachycardia, palpitations, arrhythmia, restlessness, insomnia, anxiety, tremors, psychological disturbances, and hypersensitivity reactions. Because they are minimally absorbed, topical decongestants have systemic side effects that are milder and occur less frequently compared with systemic dosage forms. Topical use may cause burning, stinging, sneezing, or local irritation. The use of topical decongestants should be limited to 3 days, since prolonged use has been associated with tachyphylaxis, rebound nasal mucosa edema, and rebound nasal congestion [14].

Since safety concerns may be present for all existing pharmacological therapies, especially for prolonged treatments, we studied the safety and effectiveness of the tested medical device.

According to the health policy that demands the assessment of patient-relevant treatment benefit for evaluation of treatments [15], symptoms have been subjectively assessed by means of VAS score. A previous study demonstrated that the use of VAS for assessing nasal obstruction appears clinically relevant, in that it allows with good reliability to quantify this symptom in the absence of rhinomanometry. In addition, it is noteworthy that VAS for rhinorrhea is also significantly associated with nasal resistance; consequently, patient's perception of nasal symptoms corresponded with objective testing [16].

The study provides evidence that there is a significant improvement in nasal and non-nasal symptoms after treatment, without any relevant side effect after 4 treatment weeks. Particularly, nasal congestion, of the relief of which is often limited, seems to benefit from the therapy, as expected on the basis of the mechanical action performed by the device.

Therefore, this first pre-post study provides the preliminary evidence that our treatment of pediatric patients affected by AR is safe and useful providing different beneficial actions on the nasal mucosa chronically inflamed without side effects.

\section{Authors' contributions/Wkład autorów}

According to order.

Conflict of interest/Konflikt interesu

None declared.
REFERENCES/PIŚ M I E N N I C T W O

[1] Asher MI, Montefort S, Bjorksten B, Lai CK, Strachan DP, Weiland SK, et al. Worldwide time trends in the prevalence of symptoms of asthma, allergic rhinoconjunctivitis, and eczema in childhood: ISAAC Phases One and Three repeat multicountry cross-sectional surveys. Lancet 2006;368: 733-743.

[2] Galant SP, Wilkinson R. Clinical prescribing of allergic rhinitis medication in the preschool and young school-age child: what are the options? BioDrugs 2001;15:453-463.

[3] Bousquet J, Khaltaev N, Cruz AA, Denburg J, Fokkens WJ, Togias A, et al. Allergic Rhinitis and its Impact on Asthma (ARIA) 2008 update (in collaboration with the World Health Organization, GA(2)LEN and AllerGen). Allergy 2008;63 (Suppl 86):8-160.

[4] Blaiss M. Current concepts and therapeutic strategies for allergic rhinitis in school-age children. Clin Ther 2004;26:1876-1889.

[5] Nathan RA. Management of patients with allergic rhinitis and asthma: literature review. South Med J 2009;102: 935-941.

[6] Bianchi ME, Manfredi AA. High-mobility group box 1 (HMGB1) protein at the crossroads between innate and adaptive immunity. Immunol Rev 2007;220:35-46.

[7] Sakamoto R, Okano M, Takena H, Ohtsuki K. Inhibitory effect of glycyrrhizin on the phosphorylation and DNAbinding abilities of high mobility group proteins 1 and 2 in vitro. Biol Pharm Bull 2001;24(8):906-911.

[8] Mollica L, De Marchis F, Spitaleri A, Dallacosta C, Pennacchini D, Zamai M, et al. Glycyrrhizin binds to high mobility group box 1 protein and inhibits its cytokine activities. Chem Biol 2007;14:431-441.

[9] Passali D, Fiorella R, Camaioni A, Villari G, Mora E, Passali GC, et al. Glucan solution nasal spray vs saline in the treatment of chronic rhinosinusitis: a multicentric double blind randomised clinical trial. Clin Ter 2007;158 (2):139-145.

[10] Ercan I, Cakir BO, Ozcelik M, Turgut S. Efficacy of Tonimer gel spray on postoperative nasal care after endonasal surgery ORL. J Otorhinolaryngol Relat Spec 2007;69(4): 203-206.

[11] Rapiejko P, Jurkiewicz D. The use of hypertonic sea-water solution in patients after surgery of the nose and paranasal sinuses. Otolaryngol Pol 2010;64(1):20-30.

[12] Psaltis AJ, Wormald PJ, Ha KR, Tan LW. Reduced levels of lactoferrin in biofilm associated chronic rhinosinusitis. Laryngoscope 2008;118(5):895-901.

[13] Eccles R. Nasal airway resistance and nasal sensation of airflow. Rhinol Suppl 1992;14:86-90.

[14] Baena-Cagnani CE. Safety and tolerability of treatments for allergic rhinitis in children. Drug Saf 2004;27:883-898.

[15] Scadding GK, Durham SR, Mirakian R, Jones NS, Leech SC, Farooque S, et al. BSACI guidelines for the management of allergic and non-allergic rhinitis. Clin Exp Allergy 2008;38:19-42.

[16] Anolik R. Desloratadine and pseudoephedrine combination therapy as a comprehensive treatment for allergic rhinitis and nasal congestion. Expert Opin Drug Metab Toxicol 2009;5:683-694. 
F URTHER REFERENCES/

LITERATURA DO D A T KOW A

Ciprandi G, Mora F, Cassano M, Gallina AM, Mora R. Visual analog scale (VAS) and nasal obstruction in persistent allergic rhinitis. Otolaryngol Head Neck Surg 2009;141:527-529

Franzke N, Schäfer I, Jost K, Blome C, Rustenbach SJ, Reich K, et al. A new instrument for the assessment of patient-defined benefit in the treatment of allergic rhinitis. Allergy 2011;66: 665-670.

Passali D, Salerni L, Passali GC, Passali FM, Bellussi L. Nasal decongestants in the treatment of chronic nasal obstruction: efficacy and safety of use. Expert Opin Drug Saf 2006;5:783-790.

van Cauwenberge P, Bachert C, Passalacqua G, Bousquet J, Canonica GW, Durham SR, et al. Consensus statement on the treatment of allergic rhinitis. European Academy of Allergology and Clinical Immunology. Allergy 2000;55:116-134. 